\title{
The Arecibo Galaxy Environments Survey-Description of the Survey and Early Results
}

\author{
R. F. Minchin ${ }^{1}$, R. Auld ${ }^{2}$, J. I. Davies ${ }^{2}$, B. Catinella ${ }^{1}$, \\ L. Cortese ${ }^{2}$, S. Linder ${ }^{3}$ E. Momjian ${ }^{1}$, E. Muller ${ }^{4}$, K. O'Neil ${ }^{5}$, \\ J. Rosenberg ${ }^{6}$, S. Sabatini ${ }^{7}$, S. E. Schneider ${ }^{8}$, M. Stage ${ }^{8}$, \\ and W. van Driel ${ }^{9}$, the AGES team
}

${ }^{1}$ Arecibo Observatory, Arecibo, United States, email: rminchin@naic.edu, ${ }^{2}$ Cardiff University, Cardiff, United Kingdom, ${ }^{3}$ University of Hamburg, Hamburg, Germany, ${ }^{4}$ Australia Telescope National Facility, Sydney, Australia, ${ }^{5}$ National Radio Astronomy Observatory, Green Bank, United States, ${ }^{6}$ Harvard Smithsonian Centre for Astrophysics, Cambridge, United States,

${ }^{7}$ Osservatorio Astronomico di Roma, Rome, Italy, ${ }^{8}$ University of Massachusetts, Amhurst, United States, ${ }^{9}$ Observatoire de Paris, Paris, France

\begin{abstract}
The Arecibo Galaxy Environments Survey (AGES) is a 2000-hour neutral hydrogen (H I) survey using the new Arecibo L-band Feed Array (ALFA) multibeam instrument at Arecibo Observatory $\dagger$. It will cover 200 square degrees of sky, sampling a range of environments from the Local Void through to the Virgo Cluster with higher sensitivity, spatial resolution and velocity resolution than previous neutral hydrogen surveys.
\end{abstract}

The first field to be covered, 5 square degrees centred on the optically-isolated galaxy NGC 1156, has revealed two possible new companions to NGC 1156, one of which may be interacting with the galaxy. This field also contains 51 definite detections in the volume beyond NGC 1156, including one behind a zone of fairly high extinction (1.4 $\mathrm{B}$ mag; Schlegel et al. 1998) to which no optical counterpart has yet been identified. A further 30 possible sources are currently being followed up at Arecibo and the GBT.

Table 1. Current status of AGES

NGC 638 (precursor field)

NGC 1156

Abell 1367 (only 20\% completed)

NGC 7332
11/2004 - 12/2004 Results published in Auld et al. (2006)

$12 / 2005-01 / 2006$ Initial results here, paper in preparation

05/2006 - 06/2006 Initial results in Cortese et al. (2007)

08/2006 - 09/2006 Currently being analysed

The status of the survey is given in Table 1 and the full fields are shown in Fig. 1 (online version only). An analysis of the noise in the NGC 1156 field is shown in Fig. 2 (online version only) and an analysis of the beam shape using continuum sources in the cube is shown in Fig. 3 (online version only). We find that the noise is Gaussian, with $\sigma=1 \mathrm{mJy}$, and that the final, gridded beam is circular with sidelobes at the $5-10 \%$ level.

\section{References}

Auld, R., Minchin, R. F., Davies, J. I. et al. 2006, MNRAS, 371, 1617.

Cortese, L., Minchin, R. F., Auld R. et al. 2007, these proceedings.

Schlegel, D. J., Finkbeiner D. P., \& Davies, M. 1998, ApJ, 500, 525.

$\dagger$ The Arecibo Observatory is part of the National Astronomy and Ionosphere Center, which is operated by Cornell University under a cooperative agreement with the NSF. 


\section{Appendix A. Online material}

AGES will address a number of scientific objectives including:

(a) the $\mathrm{H}$ I mass function in different environments;

(b) baryonic mass density;

(c) High Velocity Clouds, dwarf galaxies etc;

(d) tidal features;

(e) dynamical masses;

(f) low column density $\mathrm{H}$ i;

(g) isolated H I clouds \& dark galaxies;

(h) H I and QSO absorption features;

(i) the spatial distribution of $\mathrm{H}$ I selected galaxies.

In order to meet the scientific goals, AGES will sample the H I environment in 13 different fields spread across the sky and sampling a wide variety of environments (see Fig 1).

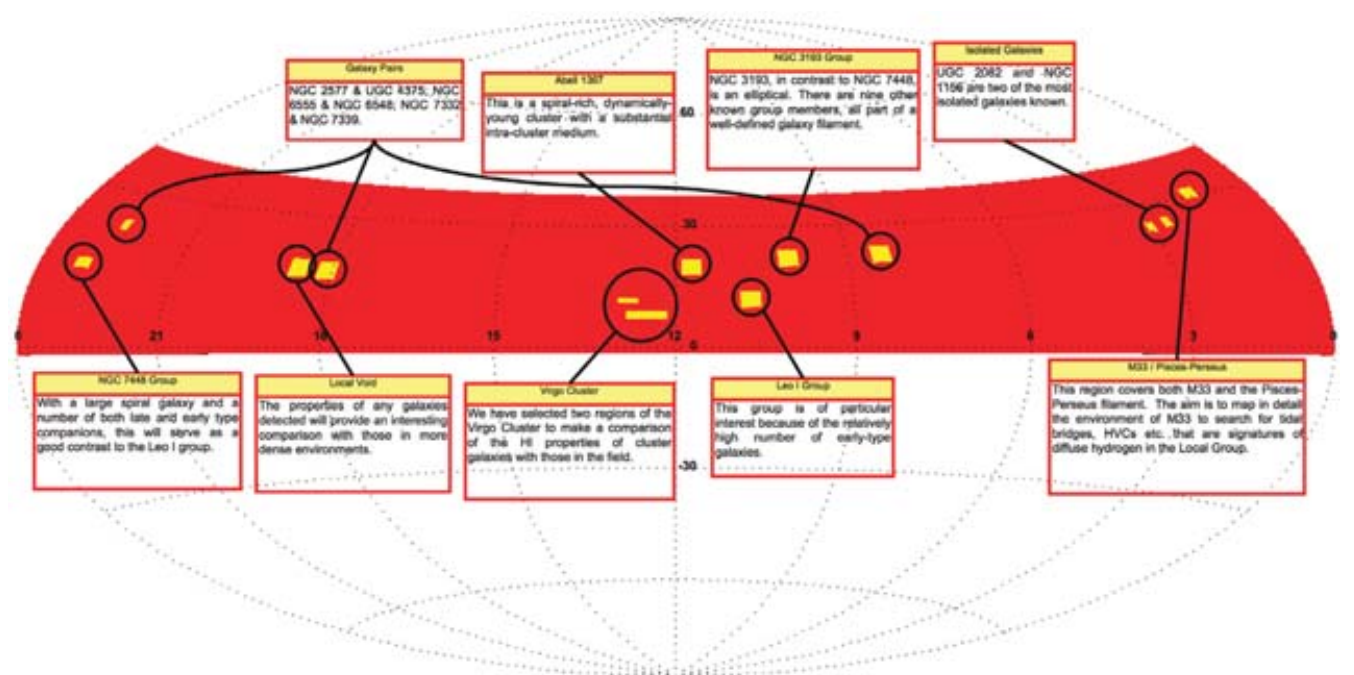

Figure 1. The AGES fields (yellow) and the Arecibo Sky (red).

We have analysed the noise levels in the NGC 1156 cube in two ways. Fig. 2a shows how the noise (measured in each RA-Dec plane) varies with velocity. There is a baseline level of around $1 \mathrm{mJy}$ (except at the edges of the cube) with spikes in the noise at certain velocities. All of these spikes can be associated with either radio frequency interference (RFI) or with real sources (see Table 2).

Table 2. Identification of noise peaks in the NGC 1156 cube

\begin{tabular}{ll}
\hline H I in the Milky Way & $0 \mathrm{~km} \mathrm{~s}^{-1}$ \\
NGC 1156 & $400 \mathrm{~km} \mathrm{~s}^{-1}$ \\
AGES J0302+2449 & $3250 \mathrm{~km} \mathrm{~s}^{-1}$ \\
Federal Aviation Authority Radar (RFI) & $5800,7000, \& 15000 \mathrm{~km} \mathrm{~s}^{-1}$ \\
Global Positioning System Satellites (RFI) & $8500 \mathrm{~km} \mathrm{~s}^{-1}$ \\
WBL 091 Group & $10500 \mathrm{~km} \mathrm{~s}^{-1}$ \\
\hline
\end{tabular}

A region free of noise spikes (identified in red in Fig. 2a) was then further analysed by looking at the distribution of the pixel values. If the noise is Gaussian, then this should 

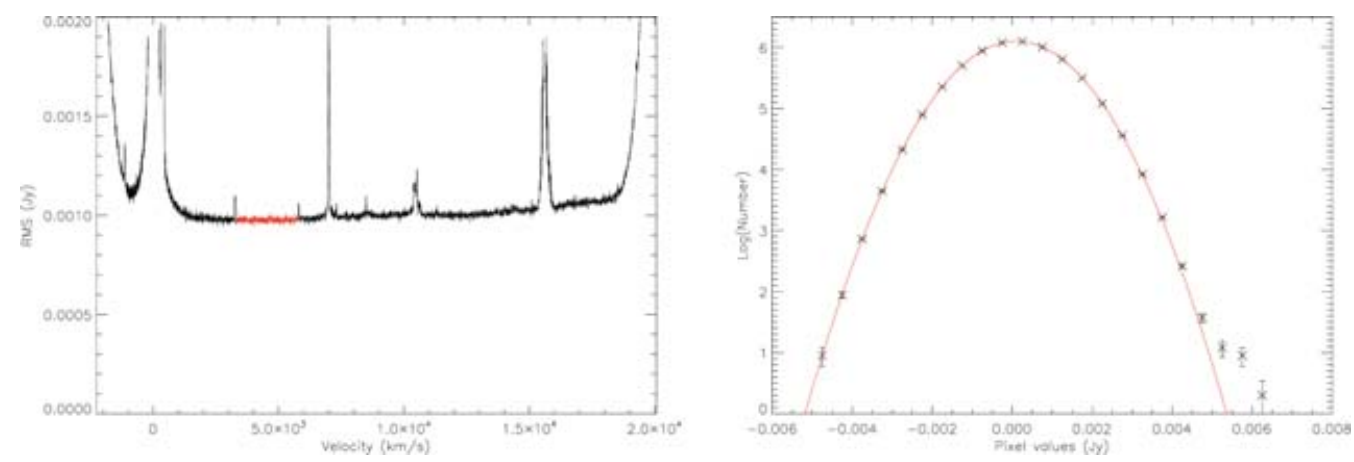

Figure 2. Left: noise in each plane along the cube. The red section marks the region used in the noise distribution analysis. Right: analysis of the noise distribution in an interference-free section of the cube; the red line shows a Gaussian distribution with $\sigma=0.98 \mathrm{mJy}$.
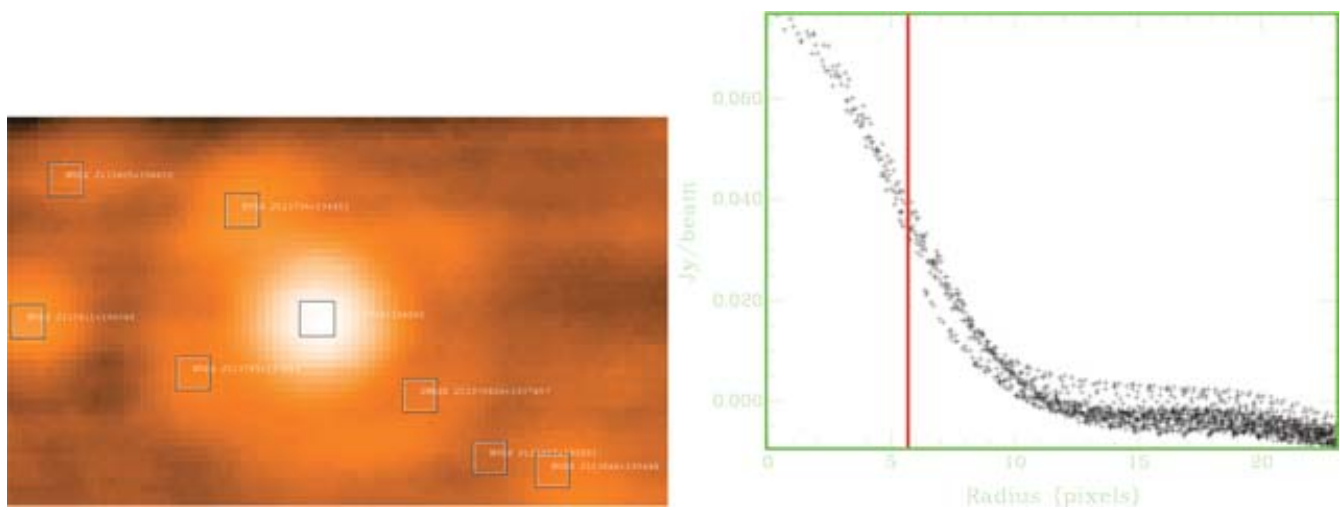

Figure 3. Left: AGES continuum map of the region around NVSS J113724+194045 in the Abell 1367 field, gridded with 0.3 arcmin pixels rather than the normal 1 arcmin pixels. This shows that the beam is fairly close to circular with a circular sidelobe pattern. Other continuum sources detected are marked with boxes and labelled. Right: radial flux distribution around NVSS J113724+194045, again with 0.3 arcmin pixels. The red vertical bar shows the size of the theoretical beam (FWHM 205 arcsec); it can be seen that this corresponds fairly well with the gridded beam. The sidelobes fall near the expected position $(1.5 \times$ FWHM from the centre $)$ and have a level of $5-10 \%$.

be a Gaussian distribution. We find (Fig. 2b) that the only deviation from the best-fit Gaussian distribution with $\sigma=0.98$ mJy comes on the positive side of the distribution at around $5 \sigma$. This is consistent with the deviation being due to real sources rather than a characteristic of the noise. We therefore conclude that the noise in the cube is Gaussian in character with a value of around $1 \mathrm{mJy}$.

We have also examined the AGES beam shape. The Arecibo beam is normally elliptical and the off-axis ALFA beams also suffer from coma. The AGES observing strategy is designed to deal with this by making a Nyquist-sampled map with every ALFA beam individually. Thus, when the observations are median combined, the variations across the beams should be removed, leaving a circular beam with symmetrical sidelobes. It can be seen from Fig. 3 that this is indeed the case. The beam appears circular with symmetrical sidelobes (Fig. 3a) and the sidelobes are at a reasonable level (5-10\%) and in the expected position. The beamsize is as expected - the gridding is handled in such a way that the final, gridded beam is little larger than the raw beam of the telescope. 